\title{
Assessing consumer-perceived food quality using conjoint analysis
}

- Le Minh Tam ${ }^{\mathrm{a}^{*}}$

- Sébasien Le ${ }^{a}$

- Nguyen Hoang Dzung

(a) Agrocampus Ouest, 65 rue de Saint-Brieuc, 35042 Rennes Cedex, France

(b) Ho Chi Minh City University of Technology, VNU-HCM, Vietnam

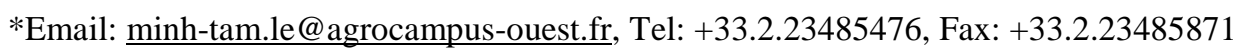

(Manuscript Received on September $30^{\text {th }}, 2014$; Manuscript Revised December $5^{\text {th }}$, 2014)

\section{ABSTRACT}

Quality is considered the most indispensable means to compete in the marketplace for all food companies. Apart from the objective quality concerning the physical - chemical microbiological characteristics, which links to the concept of food security; the subjective quality concerning the quality as perceived by consumers is utmost important since it links to the concept of consumer demand [1].

This paper proposed a new methodology which aimed to assess the subjective quality as follows: (1) to examine whether consumers could perceive and/or understand the quality criteria used by experts to assess the product quality, and (2) in case consumers could do, to investigate whether these expert criteria related to the consumer-perceived quality.

This methodology is based on the traditional conjoint analysis combining with an add-on, the

Keywords: food quality, sensory defects, holistic approach, conjoint analysis.

\section{INTRODUCTION}

The basis for all food companies is to improve product quality that insures ongoing consumer appreciation. However, it is not so easy to understand the concept of food quality. sorting task. Consumers are asked to sort 9 pictures of bananas, and then to rate the pictures according to their perception of the perceived quality presenting on the pictures. The methodology will be illustrated through a case study performed on Vietnamese consumers.

The results showed that: (1) consumers could perceive the experts' quality criteria such as bruise, shape, and color; and (2) the consumer-perceived quality related to these expert quality criteria. In addition, the results also showed that bruise was the most important attribute affect consumer appreciation of quality.

For practical application, we expected that this methodology could provide useful information about the subjective quality for those researchers who want to improve the quality based on consumer demand.
In a more general context, food quality is a multifaceted concept, which consists of four major aspects, as following: sensory attributes (either positive or defect), health (e.g., nutrition and safety), convenience (e.g., preparation, buying, storing, and 
eating), and process characteristics (e.g., organic production, animal welfare) [2]. In a simpler context in which solely the intrinsic product attributes (i.e., sensory attributes as in this context) are focused on, the food quality can be considered as overall liking of consumers [3].

Moskowitz and Krieger [4] published an article entitled "What sensory characteristics drive product quality? An assessment of individual differences". In this, their findings showed that some key sensory attributes could drive the consumer judgment of food quality: in more detail, the taste and flavor could drive the overall liking of fruit pie products. Additionally, these attributes can be used to segment consumers.

Inspired their work, but a bit far from the context of new product design decisions, our starting point aims to determine what sensory defects highly decrease the product quality. More precisely, we are in the context of food quality control, and we want to investigate the relative importance of sensory defects, imputable to each attribute levels, on the overall liking of consumers.

Why is the assessment of food quality from consumers so important? Consumers are key to driving sustainable production and play a central role in sustainable development. This alternative concept a few decades ago has become the mainstream today.

Rather than adopting the work of Moskowitz and Krieger [4] in the field of quality control, we propose in this article a new methodology for assessing the notion of quality perceived by consumers. This methodology is based on the traditional conjoint analysis combining with an add-on, the sorting task.

This minor add-on seems to be important in our context since we want to ensure that whether consumers can perceive the sensory attributes (i.e., the defects) used by the experts in the one part, and whether they can use these attributes to structure the overall liking in the other part.

This methodology consists of three main steps: constructing different product profiles from the sensory defects by means of experimental design (1), assessing the perception (2), and assessing the consumer-perceived quality (3). This methodology will be demonstrated through a "simple" case study on the quality of banana performed by Vietnamese consumers.

\section{METHODOLOGY}

\subsection{Three main steps of the methodology}

\subsubsection{Step 1: constructing the product concepts using experimental design}

An advantage of experimental design enables to investigate the structure of the profiles more systematically. By varying these profiles by an experimental design, then presenting them to subjects in a known way, researchers can understand which factors work and which do not work. Moreover, researchers can also estimate the relative contributions of these factors in the product space.

In our case study, the four factors (i.e., the expert criteria used to evaluate the visual quality of banana), such as color, shape, bruise, and cigar-end rot were chosen from the technical guidelines proposed by Dadzie and Orchard [5]. Each factor has three levels.

For varying the profiles, a full factorial design, which includes all combinations of the factor levels, could be the first-thinking solution. However, this design was not practical since the total number of combinations is large: with $3^{4}$ equals 81 combinations (i.e., the profiles) that could be constructed. This high number of profiles might lead subjects to be unduly burdened when they are asked to provide judgments on all profiles. To overcome this short-comings, using fractional factorial designs, 
which can reduce the number of products to be administered to subjects, are recommended.

Here, we constructed the profiles following a fractional factorial design - the Graeco Latin square
[6]. Following this design, there are 9 profiles (equals $3^{4-2}$ ) which could be constructed from 4 factors, each at 3 levels (cf. Table 1). These profiles are combinations of levels of the factors for which they are designed as illustrated in Fig.1.

Table 1. A $3^{4-2}$ Graeco Latin square design for 4 factors, each at 3 levels.

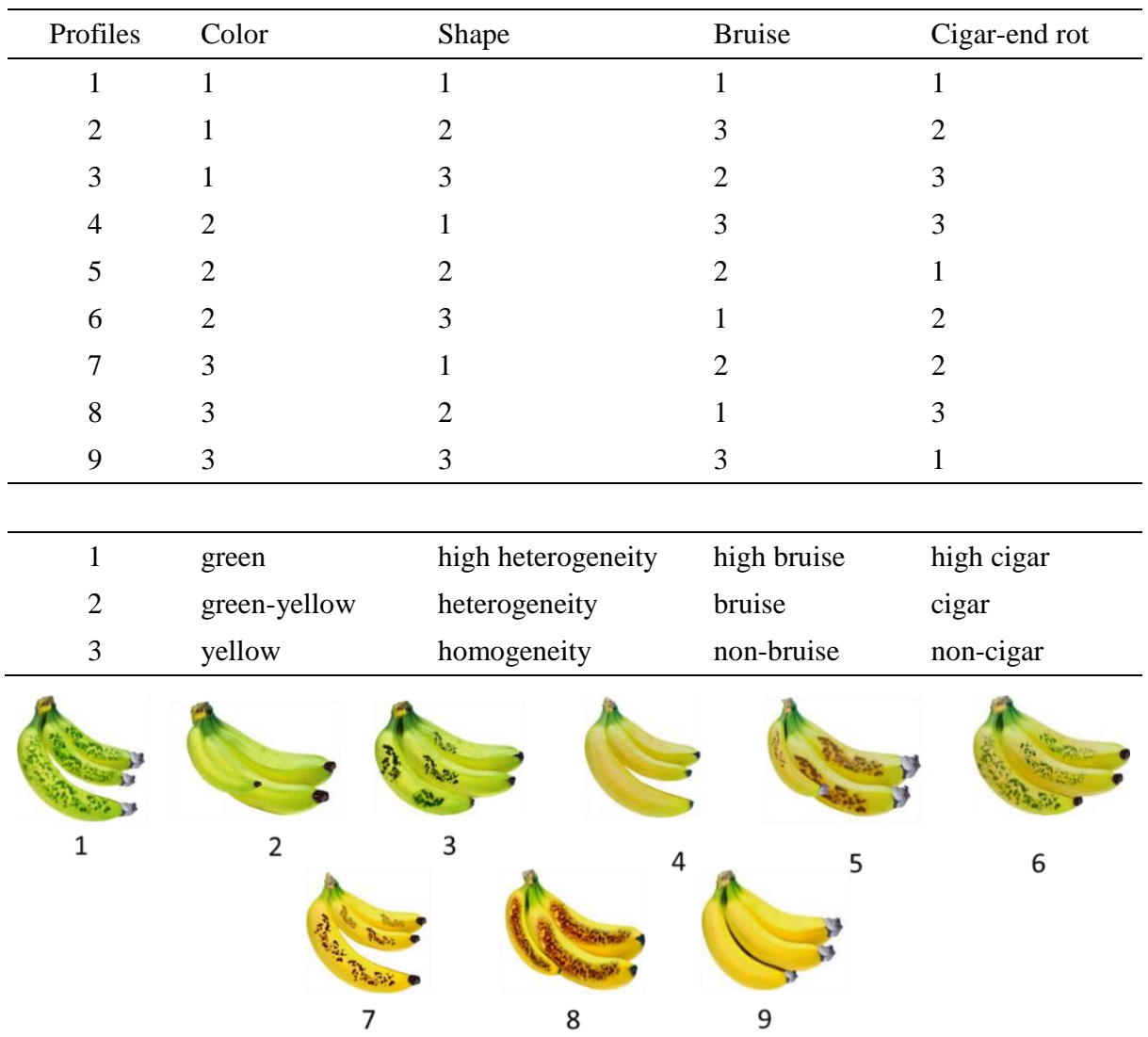

Figure 1. Profiles constructed based on the $3^{4-2}$ Graeco Latin square design.

2.1.2 Step 2: assessing consumer perception using sorting task

Most of previous conjoint applications ignore this step because the concepts of their factors and levels seem to be clearly perceived by consumers. In other words, these factors and levels could be understood without trouble, for instance: average waiting time $(10,20$, or 30 minutes) or price for a bus ticket (1, 1.5 , or 2 per hour), etc. Nevertheless, in this research context, we remark that consumers deal with the expert criteria. Thus, a natural question arises whether or not consumers can perceive the expert criteria. To do so, the sorting task was used.

The sorting aims to investigate how consumers perceive intrinsically the conceptual similarity between the profiles. In this experiment, the null hypothesis is: consumers cannot perceive the expert criteria. If this was the case, the product space could 
not be interpreted by the expert criteria. In other words, consumers might not understand the sensory defects that will be structured in the next steps. Vice versa, the alternative hypothesis is: consumers can employ the expert criteria. If this was the case, the product space could be interpreted through the expert criteria. In other words, consumers might understand the sensory defects that will be structured in the next steps.

The sorting was performed in two sessions. In the first session, each consumer was asked to sort 9 profiles into as many groups as he/she would like to, assuring that the products in the same group were perceived as the same, and the products in different groups were perceived as different. In order to get information about the sensory attributes of each group, a verbalization task was required for each consumer in the second session.

Based on the final configurations (i.e., the partitions) obtained by subjects, a data table, whose rows represent the profiles, whose columns represent the subjects, and whose intersections represent the group that a profile belongs to, was obtained.

Among the possible statistical techniques for analysing the sorting task data, e.g. MDS or DISTATIS, FAST [7] was chosen. The main advantage of FAST is that: it takes into account individual partition in the one part, and takes the same importance for each consumer when structuring the product space in the other part. Technically, FAST is a combination of MCA (which provides the consensual representation of the products, i.e. the product space) and MFA (which provides the representation of the subjects).

2.1.3 Step 3: assessing the notion of quality using conjoint analysis

Once the consumers could perceive the expert criteria (as shown in section 3.1 of this paper), we move to the next question: whether or not consumers use the expert criteria to structure the product quality. To answer this question, the traditional conjoint analysis was performed to investigate the relative importance of sensory defects on their notion of quality.

Different from the traditional conjoint analysis [8], in this experiment, all the profiles were presented simultaneously to subjects, not in a serial monadic fashion. The reason for that is because: from our point of view, once a subject evaluates all profiles simultaneously, his/her personal criteria corresponding the quality may not differ from one profile to the others. In practice, the profiles are randomly presented to subjects and they are asked to arrange these profiles on a continuous scale.

In terms of analysing the data, the attribute tradeoffs will be estimated following the part-worth model (cf. Eq.1):

$$
Y=\beta_{0}+\sum_{i, j} \beta_{i j} X_{i j}+\varepsilon \quad(\text { Eq. } 1)
$$

where $i$ denotes the number of attributes $(i=1,2$, $3,4), j$ denotes the number levels per attributes minus $1(j=1,2$ - each attribute has three levels), and $\beta$ denotes the unknown parameters (i.e., the part worth functions).

All the part worth functions are estimated by using ordinary least squares (OLS) regression method: the quality score was considered dependent variable, the levels of sensory defects were considered dummy variables. The interactions, in this model, were confounded with the error term.

\subsection{A case study and implemented software}

The proposed methodology will be demonstrated through a case study on the quality of banana, performed by 32 Vietnamese consumers. They are all students, whose age ranges from 18 to 24, at the Ho Chi Minh City University of Technology, Vietnam. 
All the subjects are asked to complete the sorting task first, then the quality rating task. In terms of collecting data, both the sorting and quality rating task were performed by using Holos environment [9]. Subjects conducted experiment using a tablet device: stimuli were displayed as icons that could be dragged with a finger. Further information about Holos could be found on http://napping.agrocampus-ouest.fr/

All statistical analyses were performed using $\mathrm{R}$ software, version 3.1.2 [10] with the package FactoMineR [11] for analysing the sorting data, and the package conjoint [12] for performing the traditional conjoint analysis.

\section{RESULTS}

\subsection{Did consumers understand the expert criteria?}

Figure 2 shows the representation of 9 product profiles obtained from MCA. This representation was the consensus over all consumers on the first factorial plane constituted by the first two dimensions of variability.
The first dimension, denoted Dim.1, explained $32.89 \%$ of total variability, whereas the second dimension, denoted Dim.2, explained $21.8 \%$ of total variability. Although the results of verbalization task were not presented in this paper, Figure 2 showed that Dim.1 opposed the products bruise on the left side to the products non-bruise on the right side of the plane, and Dim.2 opposed the products heterogeneity (levels $1 \& 2$ of the shape factor) with yellow color on the bottom left side to the products homogeneity with green color on the top left side of the plane.

These two dimensions explained a high percentage of the total variability, which indicated that there was a consensus among subjects. Interestingly, the interpretation of the two dimensions corresponded to the criteria bruise, shape and color used by experts in evaluating the quality of banana. In other words, we can conclude that consumers could perceive, and in fact they used, the expert criteria to sort the products.

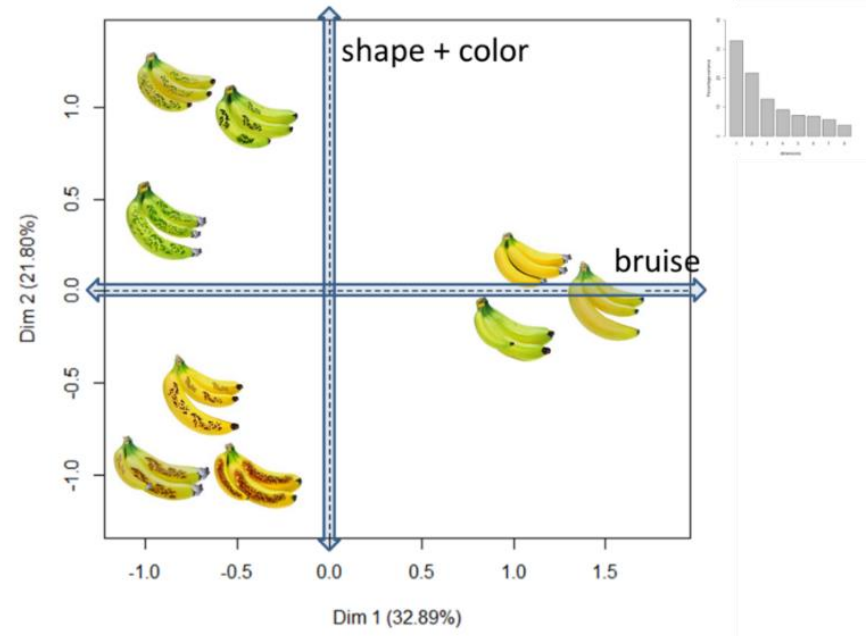

Figure 2. Consensual representation of product space perceived by consumers

3.2 How did consumers structure the relative The R-squared explains $49.22 \%$ of the variance in the importance? dependent variable (i.e., quality score), which

Table 2 shows the part worth functions, i.e. the probably be considered good fitness of the part worth parameters of the part worth model obtained by OLS. model. The results show that the part worth functions 
of the high and medium bruise levels, the level are significant different from zero.

heterogeneity level (of the shape), and the green color

Table 2. The part worth functions obtained by OLS

\begin{tabular}{|c|c|c|c|c|}
\hline \multicolumn{5}{|l|}{ Coefficients: } \\
\hline Estimate & Std. Error & t value & \multicolumn{2}{|c|}{$\operatorname{Pr}(>|t|)$} \\
\hline (Intercept) & 451,594 & 12,267 & 36,813 & $<2 \mathrm{e}-16 * * *$ \\
\hline factor $(x \$ c o l o r) 1$ & $-43,500$ & 17,349 & $-2,507$ & $0,0127 *$ \\
\hline factor $(x \$ c o 1 o r) 2$ & 16,688 & 17,349 & 0,962 & 0,3369 \\
\hline factor $(x \$$ shape $) 1$ & $-2,656$ & 17,349 & $-0,153$ & 0,8784 \\
\hline factor $(x \$$ shape $) 2$ & $-106,385$ & 17,349 & $-6,132$ & $2,95 e-09 * * *$ \\
\hline factor (x\$bruise) 1 & $-173,125$ & 17,349 & $-9,979$ & $<2 \mathrm{e}-16 * * *$ \\
\hline factor (x\$bruise)2 & $-71,240$ & 17,349 & $-4,106$ & $5,29 \mathrm{e}-05 * * *$ \\
\hline factor $(x \$ c i g a r) 1$ & $-16,500$ & 17,349 & $-0,951$ & 0,3424 \\
\hline factor $(x \$ c i g a r) 2$ & 28,146 & 17,349 & 1,622 & 0,1059 \\
\hline \multicolumn{5}{|l|}{---} \\
\hline \multicolumn{5}{|c|}{$\begin{array}{l}\text { Signif. codes: } 0 \text { ‘ } * * *, 0,001 ، * *, 0,01 ، *, 0,05 ،, 0,1 ،, 1 \\
\text { Residual standard error: } 208,2 \text { on } 279 \text { degrees of freedom }\end{array}$} \\
\hline \multicolumn{5}{|c|}{ Mu1tiple R-squared: 0.4922 , Adjusted R-squared: 0.4776} \\
\hline \multicolumn{5}{|c|}{ F-statistic: 33.8 on 8 and 279 DF, p-value: $<2,2 \mathrm{e}-16$} \\
\hline
\end{tabular}

What these results imply is that the most important defect makes banana less attractive perceived by consumers is the bruise: its high bruise level will decrease 173.1 points, and its medium bruise level will decrease 71.2 points over total of 1000 points of the quality of banana. The second important defect is the shape: its high heterogeneity level will slightly decrease 2.6 points of the quality, but its medium heterogeneity will decrease 106.3 points of the quality. Then, the third important defect is the color: its green level will decrease 43.5 points of the total quality. Finally, although the effect of the cigar-end rot defect is not significant, we can say that its high cigar level decreases 16.5 points of the total quality.

It is important to note that the two dummy variables were used to define for the three-level attribute. The dummy variables, for the third level, are automatically drop, i.e., their values are zero. The reason for that is because, from the statistical point of view, if all the dummy variables were kept, this would give a model with non-invertible $X^{\prime} X$ matrix where $X$ is the design (or model) matrix. Consequently, we could not estimate the parameters of the model because the sum of all the dummy variables is the constant predictor, which equals 1 .

However, the part worth model can be written with the intercept and all dummy variables in the case that we impose a restriction on the model, the socalled effects coding. The restriction here is: the sum of all dummy parameters is scaled to zero. This restriction might be useful if we need to rescale some the coefficients (within one factor), to make them comparable.

Tab.3 shows all the part worth functions when using effects coding (their values are rounded to one decimal place). Remarkably, the third level of

\section{Trang 26}


attributes focuses on the attractive of the quality, for instance, if the banana is at the non-bruise level, the quality will increase 244.3 points; or it is at the homogeneity level, or at the yellow color level, the quality will increase 109 or 26.8 points, respectively.
The part worth functions for each attribute can be graphically demonstrated through a bar graph (cf. Appendix).

For a better interpretation, this fitted model can be rewritten with all the part worth functions as follows:

$Y=451.5-43.5 \times$ green $+16.6 \times$ green.yellow $+26.8 \times$ yellow $-2.6 \times$ high.heterogeneity $-106.3 \times$ heterogeneity $+109.0 \times$ homogeneity $-173.1 \times$ high.bruise $-71.2 \times$ med.bruise $+244.3 \times$ bruise $-16.5 \times$ high.cigar $+28 \times$ med.cigar $-11.6 \times$ low.cigar

Figure 3 shows the relative importance of attributes in a total utility function. This relative measure was obtained by rescaling these measures such that they all add to $100 \%$. This result shows that bruise is the most important sensory defect, which contributes $45.62 \%$ of total quality. Then, the second important defect is the shape, which contributes $23.45 \%$ of total quality. The color and the cigar-end rot contribute respectively $17.68 \%$ and $13.25 \%$ of total quality.

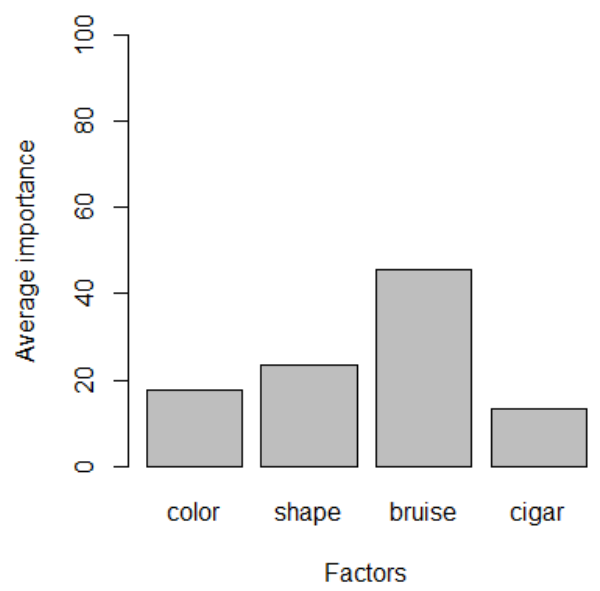

Figure 3. Relative importance of sensory defects on the quality of banana

Table 3. The part worth functions of all levels

\begin{tabular}{lll}
\hline Leve1s & $P-W$ & function.values \\
\hline 1 & intercept & 451,5 \\
2 & green & $-43,5$ \\
3 & green-ye11ow & 16,6 \\
4 & yel1ow & 26,8 \\
5 & high.heterogeneity & $-2,6$ \\
6 & heterogeneity & $-106,3$ \\
7 & homogeneity & 109,0
\end{tabular}




\begin{tabular}{llll}
\hline 8 & high.bruise & $-173,1$ \\
9 & bruise & $-71,2$ \\
10 & non.bruise & 244,3 \\
11 & high.cigar & $-16,5$ \\
12 & med.cigar & 28,1 \\
13 & non.cigar & $-11,6$ \\
\hline
\end{tabular}

\section{CONCLUSIONS}

This paper shows an evidence that the two most and about the part worth model, which aids in common beliefs about consumers and consumerperceived quality, at least in the context of quality control, are wrong:

Consumers cannot perceived the quality criteria used by experts.

Consumer perceived quality does not have structure and it does not related to the expert criteria.

Besides, the proposed methodology provides valuable information about key sensory attributes driven consumer-perceived quality in the one part; interpreting the relative contribution of each attribute as well as its levels affect the quality in the other part.

In terms of the data collection, the two experiments of this methodology were performed by using Holos environment. By observing subjects and doing surveys, subjects showed great interest in using tablet: it is intuitive, easy, and entertaining.

We expected that this methodology could be a useful tool for practitioners and researchers who want to study the consumer-perceived quality in order to improve the total quality of product. 
APPENDIX

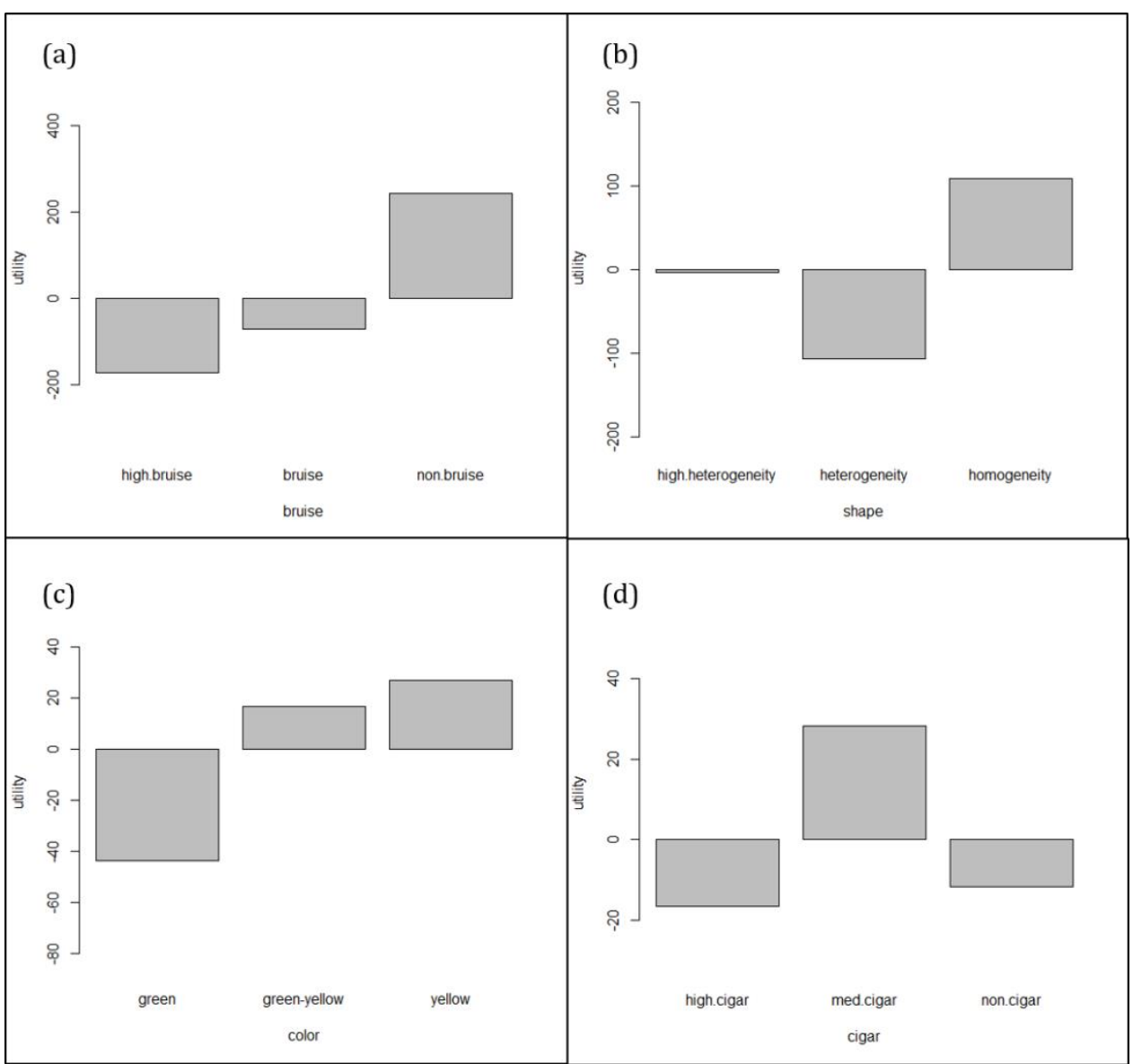

Figure A1. The part worth function for the quality of banana. bruise, (b) shape, (c) color, and (d) cigar. 


\section{Tìm hiểu chất lượng thực phẩm bằng phương pháp phân tích đánh đổi}

- Lê Minh Tâma ${ }^{*}$

- Sébastien Lêa

- Nguyễn Hoàng Dũng ${ }^{b}$

(a) Agrocampus Ouest, 65 rue de Saint-Brieuc, 35042 Rennes Cedex, France

(b) Trường Đại học Bách khoa, ĐHQG-HCM

\section{TÓM TÁT}

Có hai câu hỏi nghiên cứu mà chúng tôi nhắm đến: (1) liệu người tiêu dùng có thể cảm nhận được các tiêu chuẩn chất lượng được sử dụng bởi các chuyên gia? Nếu có, (2) liệu người tiêu dùng có sử dụng các tiêu chuẩn này để đánh giá chất lượng sản phẩm?

Bên cạnh việc trả lời hai câu hỏi nghiên cứu trên, một cách tổng quát hơn, bài báo này sẽ trình bày một phương pháp tiếp cận mới phương pháp tiếp cận tổng thể - chất lượng thực phẩm theo quan điểm người tiêu dùng. Phương pháp này là sự kết hợp giữa: phân nhóm tự do (sorting task) và phân tích đánh đổi (có một tên gọi khác là phân tích kết hợp conjoint analysis). Phương pháp sẽ được minh

\section{REFERENCE}

[1]. M. M. Pagliuca and D. Scarpato, "Food quality, consumer perception and preferences: an analysis on olive oil," Electronic Journal of Applied Statistical Analysis, vol. 4, pp. 215226, 2011.

[2]. K. G. Grunert, "Current issues in the understanding of consumer food choice," Trends in Food Science \& Technology, vol. 13, pp. 275-285, 2002. họa để tìm hiểu chất lượng sản phẩm chuối trên đối tượng là người tiêu dùng Việt Nam.

Kết quả chỉ ra rằng: (1) người tiêu dùng có thể cảm nhận được các tiêu chuẩn chất lượng được sử dụng bởi chuyên gia như vết thâm, độ đồng đều kích thước quả và màu sắc; hơn nữa (2) các tiêu chuẩn chất lượng này có mối liên hệ chặt chẽ với chất lượng sản phẩm. Bên cạnh đó, kết quả của nghiên cứu cũng chỉ ra rằng: đối với chuối, vết thâm được đánh giá là "Iỗi" nghiêm trọng nhất làm giảm chất lượng sản phẩm.

Chúng tôi hy vọng phương pháp được đề nghị trong nghiên cứu sẽ là công cu hữu ích để doanh nghiệp có thể nâng cao chất lượng sản phẩm đáp ứng nhu cầu người tiêu dùng.

[3]. H. R. Moskowitz, "Food quality: conceptual and sensory aspects," Food Quality and Preference, vol. 6, pp. 157-162, 1995.

[4]. H. Moskowitz and B. Krieger, "What sensory characteristics drive product quality? An assessment of individual differences," Journal of Sensory Studies, vol. 8, pp. 271-282, 1993.

[5]. B. K. Dadzie and J. E. Orchard, Routine postharvest screening of banana/plantain hybrids: criteria and methods vol. 2: Bioversity International, 1997. 
[6]. T. Lorenzen and V. Anderson, Design of experiments: a no-name approach: CRC Press, 1993.

[7]. M. Cadoret, S. Lê, and J. Pagès, "A Factorial Approach for Sorting Task data (FAST)," Food quality and preference, vol. 20, pp. 410-417, 2009.

[8]. V. R. Rao, "Applied Conjoint Analysis," p. 389, 2014.

[9]. M. T. Lê and S. Lê, "Holos: a collaborative environment for holistic approaches," Poster Presented at Sensometrics Chicago, IL., 2014.

[10]. R. C. Team, "R: A Language and Environment for Statistical Computing. R Foundation for
Statistical Computing, Vienna, Austria, 2012," ISBN 3-900051-07-02012.

[11]. F. Husson, J. Josse, S. Le, and J. Mazet, "FactoMineR: multivariate exploratory data analysis and data mining with $\mathrm{R}, " R$ package version, vol. 1, pp. 102-123, 2013.

[12]. A. Bak and T. Bartlomowicz, "Conjoint analysis method and its implementation in conjoint R package," In: Pociecha J., Decker R. (Eds.), Data analysis methods and its applications, C.H. Beck, pp. 239-248, 2012. 